\title{
Spinodal and thermodynamic limit of superheat of gold
}

\author{
Marimuthu Sivakumar and Ramasamy Balasubramanian* \\ Department of Physics, Arignar Anna Government Arts College, Namakkal 637 002, Tamilnadu, India
}

\begin{abstract}
A four-parameter generalized Berthelot equation of state has been employed to determine the spinodal and the thermodynamic limit of superheat of liquid gold. It is established that gold obeys the two-parameter law of corresponding states. It is also established that the new parameters introduced in the attractive term of the equation of state are thermodynamic similarity parameters. It is shown that liquid gold can be superheated to a temperature $6719 \mathrm{~K}$. That is, liquid gold, under rapid heating, can be superheated to temperature $3476 \mathrm{~K}$ above the boiling temperature without undergoing liquid-vapour phase transition. Above this temperature, liquid gold will undergo explosive boiling owing to homogeneous nucleation.
\end{abstract}

Received 3 August 2020 Accepted 10 December 2020

*For correspondence: drrbala@yahoo.com

Contact us: sciencevision@outlook.com

Keywords: Corresponding states, equation of state, gold, superheat, spinodal.

\section{Introduction}

The nanoparticles of noble metals exhibit physical properties that make them unique for scientific and technological applications: electronics, catalysis, biotechnology, spectroscopy, etc. The thermochemical properties of the compounds and inter metallic phases of gold have been well investigated. One of the functional tasks of statistical mechanics and thermodynamics is to find the equations of state of substances. Having accurate equations of state for the substances is important in the design of several industrial processes and for precise calculations describing a variety of thermodynamic processes. Many modifications of the known equations of state have been proposed. ${ }^{1-7}$

These modifications result in the generalization of the known equations of state with a numerical stability and ability to describe the thermodynamic properties of a wide range of technically important substances used in industries. To improve the accuracy of an equation of state usually its attractive term and the repulsive term are modified. In some cases, the equation - of - state parameters dependence on temperature is assumed. Laser ablation and explosion of wires are two techniques of producing gold nano particles. In these processes, gold is rapidly heated. Under these conditions, gold is superheated to temperature, much above its normal boiling temperature. The spinodal, on the phase diagram, is the stability boundary. Beyond this boundary, gold undergoes explosive boiling. To understand the mechanism of superheating and explosive boiling, the knowledge of the spinodal and the thermodynamic limit of superheat is required. The present work, based on a new four-parameter generalized Berthelot equation of state, deals with the determination of the spinodal and the thermodynamic limit of superheat of gold. Considering the difficulties in obtaining reliable experimental data on the spinodal and superheating of gold, this work acquires scientific and 
technological significance.

\section{Generalized Berthelot Equation of State}

To improve the accuracy of the known Berthelot equation of state, an improvement is proposed by introducing the parameters $m$ and $n$ in its attractive term. Such a generalized Berthelot equation of state for one mole of substance has the form:

$$
P=\frac{R T}{V-b}-\frac{a}{T^{m} V^{n}}
$$

Where $P$ - pressure, $V$ - molar volume, $T$ temperature, $R$ - universal gas constant, $a, b, m$ and $n$ are substance-specific parameters.

The liquid-vapour critical point conditions are

$$
\left(\frac{\partial P}{\partial V}\right)_{T_{c}}=0,\left(\frac{\partial^{2} P}{\partial V^{2}}\right)_{T_{c}}=0
$$

Application of the critical - point conditions to the equation of state given by Eq. (1) gives the critical volume, critical temperature and critical pressure as

$$
\begin{aligned}
& V_{c}=\left(\frac{n+1}{n-1}\right) b \\
& T_{c}=\left[\frac{4 n a}{R(n+1)^{2}}\left(\frac{n-1}{b(n+1)}\right)^{n-1}\right]^{\frac{1}{m+1}} \\
& P_{c}=\left(\frac{n-1}{2 n}\right)\left[\frac{n a R^{m}(n-1)^{m+n}}{2^{m-1} b^{m+n}(n+1)^{n+1}}\right]^{\frac{1}{m+1}}
\end{aligned}
$$

The critical compressibility factor is then given by

$$
Z_{c} \equiv \frac{P_{c} V_{c}}{R T_{c}}=\frac{n^{2}-1}{4 n}
$$

Taking into account Eqs. (2)-(5), we may write Eq. (1) in terms of reduced variables

$$
\begin{aligned}
& P^{*}=P / P_{c}, V^{*}=V / V_{c}, T^{*}=T / T_{c} \text { as } \\
& P^{*}=\frac{1}{n-1}\left[\frac{4 n T^{*}}{(n+1) V^{*}-(n-1)}-\frac{n+1}{T^{* m} V^{* n}}\right]
\end{aligned}
$$

The reduced equation of state given by Eq. (6) represents the two - parameter law of corresponding states with the thermodynamic similarity parameters $m$ and $n$. That is, substances obeying the generalized Berthelot equation of state, with the same values of parameters $m$ and $n$ are thermodynamically similar. Such substances have similar intermolecular force characteristics. It follows from Eqs. (2) and (5) that parameter $V_{d} b$ or $Z_{c}$ can also be used as the thermodynamic similarity parameter instead of the parameter $n$.

\section{Determination of the Equation-of-state}

\section{Parameters}

The parameters of the generalized Berthelot equation of state may be determined through the critical-point parameters of substances. The use of the critical-point parameters in determining the parameters of the equation of state will improve the ability of the equation of state to determine the high -temperature properties of substances.

Eq. (5) may be rearranged as

$$
n^{2}-4 n Z_{c}-1=0
$$

Eq. (7) is a quadratic equation having two solutions for $n$. The parameter $n$ cannot have a negative value. Hence, we have

$$
n=2 Z_{c}+\sqrt{4 Z_{c}^{2}+1}
$$

Riedel's parameter is defined as

$$
\alpha_{R}=\left(\frac{\partial P^{*}}{\partial T^{*}}\right)_{V^{*}}, \text { at critical point }
$$

From Eqs. (6) and (9), we get

$$
\alpha_{R}=\frac{2 n+m(n+1)}{n-1}
$$

Eq. (10) may be rearranged to get the expression for $m$ as

$$
m=\frac{(n-1) \alpha_{R}-2 n}{n+1}
$$

Eq. (2) may be rearranged to get the expression for $b$ as

$$
b=\left(\frac{n-1}{n+1}\right) V_{c}
$$

Eq. (3) may be rearranged to get the expression for $a$ as

$$
a=\frac{(n+1)^{2} R V_{c}^{n-1} T_{c}^{m+1}}{4 n}
$$

Eqs. (8)-(13) enable one to determine the parameters $a, b, m$ and $n$ through the critical-point parameters and the Riedel's parameter of substances. 


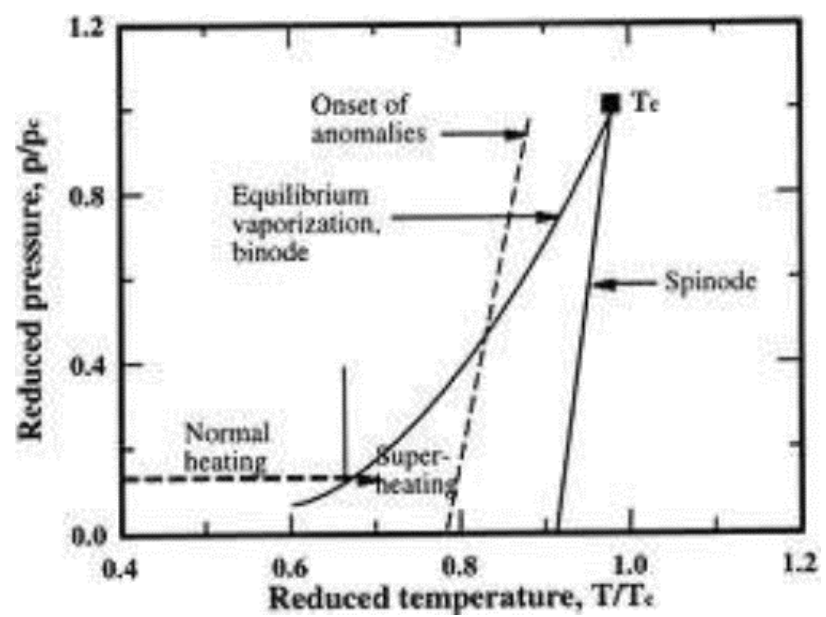

Figure 1 | Schematic phase diagram of substances.

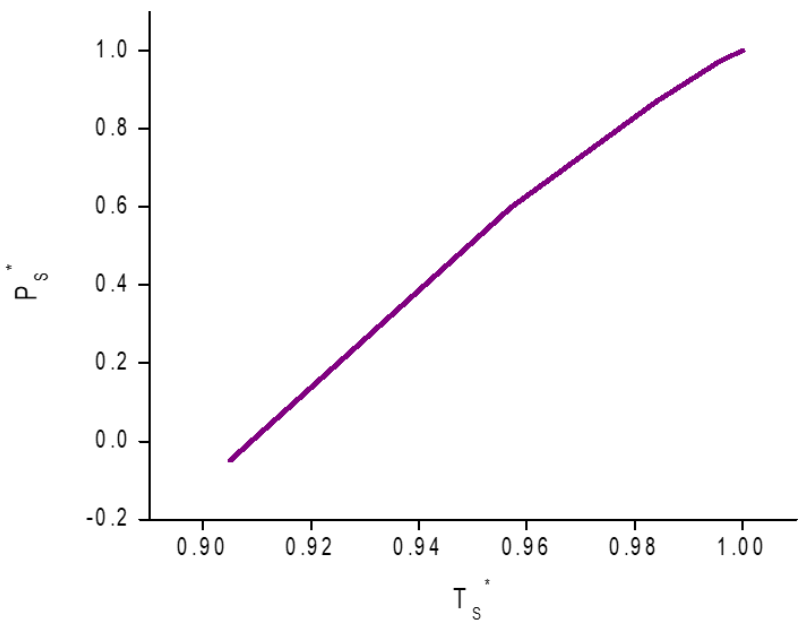

Figure 2 | Spinodal of gold.

Table 1 | Parameters of the generalized Berthelot equation of state.

\begin{tabular}{|c|c|c|c|c|}
\hline Metal & $\underset{\mathrm{Jkg}^{-1} \mathrm{~K}^{-1} \mathrm{~m}^{3} \mathrm{~mol}^{-1}}{\stackrel{1}{1}}$ & $\begin{array}{c}b \\
10^{-5} \mathrm{~m}^{3} \mathrm{~mol}^{-1}\end{array}$ & $m$ & $n$ \\
\hline Gold & 1849719.9 & 0.5380 & 0.183 & 1.533 \\
\hline
\end{tabular}

Table 2 | Thermodynamic limit of superheat of gold.

\begin{tabular}{|c|c|c|c|c|}
\hline Metal & $T_{s, 0}^{*}$ & $\begin{array}{c}T_{s, 0} \\
\mathbf{K}\end{array}$ & $V_{s, 0}^{*}$ & $\begin{array}{c}V_{s, 0} \\
10^{-5} \mathrm{~m}^{3} / \text { mole }\end{array}$ \\
\hline Gold & 0.908 & 6719 & 0.605 & 1.5470 \\
\hline
\end{tabular}

Table 3 | Spinodal of gold.

\begin{tabular}{ccc}
$V_{s}^{*}$ & $T_{s}^{*}$ & $P_{s}^{*}$ \\
0.2 & 0.018 & -127.100 \\
0.3 & 0.332 & -20.362 \\
0.4 & 0.637 & -5.750 \\
0.5 & 0.809 & -1.610 \\
0.6 & 0.905 & -0.048 \\
0.7 & 0.957 & 0.598 \\
0.8 & 0.984 & 0.870 \\
0.9 & 0.996 & 0.975 \\
1.0 & 1.000 & 1.000 \\
\hline
\end{tabular}




\section{Spinodal and Thermodynamic Limit of Superheat}

The knowledge of the spinodal, a characteristic curve on the phase diagram (Fig. 1), is essential in describing the properties of substances in the critical and in the metastable states with decreased thermodynamic stability. The spinodal defines the thermodynamic stability boundary of the phase envelope. Inside the spinodal envelope, a system is unstable. Above $T_{c}$, the liquid phase becomes indistinguishable from vapour phase, ${ }^{8-14}$ as the free energy barrier separating them vanishes.

Moreover, the critical point lies on both the spinodal and binodal lines. The liquid can be superheated to the temperatures well above their equilibrium boiling temperatures. Such nonequilibrium metastable states can exist only during finite time interval because of spontaneous development of growing vapour bubbles.

The spinodal is, defined by the condition,

$$
-\left(\frac{\partial P}{\partial V}\right)_{T}=0
$$

Liquids remaining in the liquid state above the boiling temperature in the ambient pressure are said to be superheated. These liquids are in metastable state in a thermodynamic sense. A liquid cannot be superheated up to its critical temperature. There is a limit to the maximum attainable temperature for any liquid without boiling. This heating limit is called the maximum attainable superheat or the thermodynamic limit of superheat. ${ }^{15,16}$

The thermodynamic limit of superheat is given by the conditions,

$$
-\left(\frac{\partial P^{*}}{\partial V^{*}}\right)_{T^{*}}=0, P^{*}=0
$$

Application of the condition given by Eq.(14)to Eq.(6) gives the spinodal in $V^{*}, T^{*}$ coordinates and in $P^{*}, V^{*}$ coordinates as

$$
\begin{aligned}
& T_{s}^{*}=\left[\frac{\left[(n+1) V_{s}^{*}-(n-1)\right]^{2}}{4 V_{s}^{* n+1}}\right]^{\frac{1}{m+1}} \\
& P_{s}^{*}=\frac{4^{\frac{m}{m+1}}\left[(n+1) V_{s}^{*}-n\right]}{V_{s}^{* \frac{n+1}{m+1}}\left[(n+1) V_{s}^{*}-(n-1)\right]^{\frac{2 m}{m+1}}}
\end{aligned}
$$

As seen from Fig. 1, with the decrease in pressure, the difference between the temperature of superheat and the equilibrium boiling temperature at a given pressure, increases. At zero pressure, the difference between the temperature of superheat and the equilibrium boiling temperature at a given pressure attains the maximum. The maximum value of the difference between the temperature of superheat and the equilibrium boiling temperature, under zero pressure, is a characteristic thermodynamic parameter of liquids called the thermodynamic limit of superheat. Hence, in this work, the thermodynamic limit of superheat under zero pressure is determined.

Under zero pressure, i.e $P_{s}^{*}=0$, Eq. (17) gives

$$
\begin{aligned}
& \frac{4^{\frac{m}{m+1}}\left[(n+1) V_{s}^{*}-n\right]}{V_{s}^{* \frac{n+1}{m+1}}\left[(n+1) V_{s}^{*}-(n-1)\right]^{2 m}}=0 \\
& {\left[(n+1) V_{s}^{*+1}-(n-1)\right]^{2}=0} \\
& V_{s, 0}^{*}=\frac{n}{n+1}
\end{aligned}
$$

Substituting Eq. (18) into Eq. (16), we get

$$
T_{s, 0}^{*}=\frac{1}{4^{\frac{1}{m+1}}}\left(\frac{n+1}{n}\right)^{\frac{n+1}{m+1}}
$$

Eqs. (18) and (19) give the expressions for the parameters of the thermodynamic limit of superheat.

By substituting the values of thermodynamic similarity parameter $m$ and $n$ of gold, the reduced volume of liquid gold at the maximum attainable superheat under zero pressure and maximum attainable reduced temperature of superheat at zero pressure are estimated through Eqs. (18) and (19). The obtained results are presented in Table 2 .

With the obtained values of the parameters $m$ and $n$ (Table 1), the spinodal of gold is determined through Eqs. (16) and (17). The results are presented in Table 3. Moreover, the spinodal of gold is plotted in Fig. 2.

\section{Results and Discussion}

As seen from Table 1, the value of the parameter $n$ is greater than that of the parameter $m$. That is, the attractive term in the generalized Berthelot equation of state has a stronger dependence on volume than on the temperature. The spinodal (stability boundary on the phase diagram) of gold and the thermodynamic limit of superheat of gold have liquid been determined. As seen from Table 2, the liquid gold, under zero pressure, can be superheated to a temperature $0.908 \mathrm{~T}_{\mathrm{c}}$. This value agrees with the experimental value. ${ }^{22}$ That is, liquid gold can be superheated to a temperature $3476 \mathrm{~K}$ above its normal boiling temperature. At the thermodynamic limit of superheat, the volume of the liquid gold is $1.547 \times 10^{-5} \mathrm{~m}^{3} \mathrm{~mol}^{-1}$. Above this 
temperature, liquid gold will undergo explosive boiling owing to homogeneous nucleation.

\section{Conclusion}

The spinodal (stability boundary on the phase diagram) of gold and the thermodynamic limit of superheat of liquid gold have been determined. It has been established that the liquid gold, under zero pressure, can be superheated to a temperature $0.908 \mathrm{~T}_{\mathrm{c}}$. This value agrees with the experimental value. That is, liquid gold can be superheated to a temperature $3476 \mathrm{~K}$ above its normal boiling temperature. At the thermodynamic limit of superheat, the volume of the liquid gold is $1.547 \mathrm{X}$ $10^{-5} \mathrm{~m}^{3} \mathrm{~mol}^{-1}$. Above this temperature, liquid gold will undergo explosive boiling owing to homogeneous nucleation.

\section{Conflict of interest}

The authors declared no conflict of interest.

\section{References}

1. Martynyuk M.M. (1999). Journal of Engineering Physics and Temperature, 72, 4 .

2. Martynyuk M.M., Balasubramanian R. (1995). International Journal of Thermophysics, 16, 2.

3. Balasubramanian R., Menaka S. (2016). International Journal of Science and Research, 5, 634636.

4. Balasubramanian R., Kamala R. (2016). Open Science Journal of Modern Physics, 3(1), 1-4.

5. Balasubaramanian R., Gunavathi K. (2014). Jegan R, Roobanguru D. Open Journal of Modern Physics, $1,54-60$.

6. Sobko VAA. (2014). Journal of Physical Science, 4(8).

7. Jaime Wisniak .( 2010). Education. Quím., 21(2), 155 -162 .
8. Insepov Z. (2007). American Nuclear Society, LaGrange Park, California, Illinois.

9. Balasubramanian R. (2019). American Journal of Mathematical Science Application. 7(3), 60-64.

10. Ermakov G.V., Lipnyagov E.V., Perminov S.A., Gurashkin L. (2009). Journal of Chemical Physics, 131, 031102.

11. Petrovyu V., Inogamov N.A., Anisimor S.I., Khishchenko K.V. (2015). Journal of Physics, 65316.

12. Balasubramanian R., Arul C. (2017). International Journal of Science and Research, 6, 2315-2319.

13. Zamiri R., Zakaria A., Ahanger H.A., Sadrolhosseini A.R., Mahdi M.A. (2010). International Journal Molecular Science, 11, 47644770.

14. Chen X., Munjiza A., Zhang K., Wen D. (2014). Journal of Physics Chemistry C, 118, 1285-1293.

15. Arblaster J.W. (2016). Journal of Phase Equilibria and Diffusion, 1547-7037.

16. Zhang Y., Evans J. R. G., Yang S. (2011). Journal Chemistry Engineering Data, 56, 328-337.

17. HoKhacHieu, Nguyen Ngoc Ha. (2013). AIP Advances, 3, 112125.

18. Khomkin A.L., Shumikhin A.S. (2016). Joint Instant for High Temperature of RAS, Moscow (Russia).

19. Boboridis K, Pottalacher G, Jager H. (1999). International Journal Thermophysics, 20, 1289-1297.

20. Singh J.K., Adhikari J., Kwak S.K. (2006). Fluid Phase Equilibria, 248, 1-6.

21. Masanori Matsui. (2010). Journal of Physics, 215, 012197.

22. Martynyuk MM. (1992). Thermochimica Acta, 206, 55-60. 